Cinémas

Revue d'études cinématographiques

Journal of Film Studies

\title{
On the road to Kandahar \\ On the Road to Kandahar
}

\section{Pierre Kadi Sossou}

Volume 18, numéro 2-3, printemps 2008

Le road movie interculturel

URI : https://id.erudit.org/iderudit/018557ar

DOI : https://doi.org/10.7202/018557ar

Aller au sommaire du numéro

Éditeur(s)

Cinémas

ISSN

1181-6945 (imprimé)

1705-6500 (numérique)

Découvrir la revue

Citer cet article

Kadi Sossou, P. (2008). On the road to Kandahar. Cinémas, 18(2-3), 165-190.

https://doi.org/10.7202/018557ar
Résumé de l'article

Voyage, rencontre de cultures, double identité du personnage Nafas constituent des éléments qui fondent Kandahar, le film du réalisateur iranien Mohsen Makhmalbaf (2001), comme road movie interculturel. Prenant pour prétexte la quête d'une soeur à sauver à Kandahar, le film fait de la narration de la route un expédient pour promener le spectateur dans les méandres de l'Afghanistan des Talibans. Sur cette route qui oriente vers les clivages entre hommes et femmes afghans, Nafas n'a de cesse d'enlever et de mettre son voile (burqa), donnant ainsi lieu de s'interroger sur la posture du dévoilement, au sens propre - enlever la burqa - comme au figuré - révéler au monde les conditions de vie des Afghans. Ce geste de dévoilement se veut symbole d'une liberté qui s'oppose aux canons du fondamentalisme religieux. 


\section{On the road to Kandahar}

\section{Pierre Kadi Sossou}

\section{RÉSUMÉ}

Voyage, rencontre de cultures, double identité du personnage Nafas constituent des éléments qui fondent Kandahar, le film du réalisateur iranien Mohsen Makhmalbaf (2001), comme road movie interculturel. Prenant pour prétexte la quête d'une sœur à sauver à Kandahar, le film fait de la narration de la route un expédient pour promener le spectateur dans les méandres de l'Afghanistan des Talibans. Sur cette route qui oriente vers les clivages entre hommes et femmes afghans, Nafas n'a de cesse d'enlever et de mettre son voile (burqa), donnant ainsi lieu de s'interroger sur la posture du dévoilement, au sens propre enlever la burqa - comme au figuré - révéler au monde les conditions de vie des Afghans. Ce geste de dévoilement se veut symbole d'une liberté qui s'oppose aux canons du fondamentalisme religieux.

\section{For English abstract, see end of article}

À sa sortie au Festival de Cannes en 2001, Kandahar - film documentaire du réalisateur iranien Mohsen Makhmalbaf remporte un succès fulgurant: Prix du Public (Cannes, 2001), Médaille d'Or Fellini (Unesco, 2001), Grand Prix du Jury International Ecuménique (Ocic-Interfilm, 2001) et bien d'autres. Kandahar est, comme le précise Nalofer Pazira (2001) ${ }^{1}$ dans une interview, "une collection d'histoires sur la pauvreté et la tragédie causées par la guerre en Afghanistan ». L'histoire extradiégétique prise en charge ici par le médium cinéma est simple: Nalofer Pazira, jeune femme afghane réfugiée au Canada, reçoit « une lettre désespérée de son amie qui voulait se suicider à cause des conditions de vie trop rudes à Kandahar " (Haghighat 2001). Dans le rôle de cette jeune afghane, Nafas quitte le Canada en toute hâte pour aller trouver et secourir cette amie devenue sœur dans l'univers diégétique. Toute l'intrigue tient en la recherche de cette sœur à sauver, et la route 
devient lieu de quête, lieu de rencontres aussi. Rencontre d'abord d'une famille afghane de retour d'Iran, puis rencontre de Khak, un jeune Afghan de douze ans, réfugié - comme de nombreux figurants dans le film — avec sa mère à Niatak, un village à la frontière iranienne. Rencontre ensuite de Tahib Sahid, un Américain noir devenu médecin de village à Niatak. La boucle des rencontres importantes sera bouclée par celle de Hâyat, un Afghan qui fera avec Nafas la dernière tranche du parcours jusqu’à un poste de sécurité érigé par les Talibans.

En fixant le regard sur l'itinéraire de Nafas, sur ses moyens (technique et animal) de locomotion et sur les rencontres culturelles dans le film, je formule l'hypothèse que la route qu'emprunte Nafas contribue à faire de Kandahar un road movie interculturel. En effet, outre la forme de rencontre culturelle manifeste dans des séquences où des personnages de divers horizons culturels sont appelés à interagir, au moins deux éléments du film permettent de problématiser l'interculturel: une rencontre culturelle s'opère dans le personnage de Nafas, personnage à l'identité double qui assume sa culture afghane (orientale) et sa culture canadienne (occidentale). Un second élément, le plus important de ceux qui posent la rencontre culturelle comme l'un des enjeux qui traversent le film, est la question du voile. Le réalisateur joue en effet sur les images de voilement et de dévoilement pour atteindre l'un des objectifs explicites de ce voyage cinématographique: faire la lumière sur ce que cache le voile, ici la burqa ${ }^{2}$, vêtement recouvrant entièrement certaines femmes afghanes. L'opposition entre voilement et dévoilement étant au cour du périple de Nafas, je vais, avant de situer aux lecteurs les moyens cinématographiques mis en œuvre pour réaliser le conflit entre hijâb et kashf (entendre voilement-dévoilement), interroger l'interculturalité dans le film, tout en établissant en quoi cette dernière se rapporte au genre du road movie ${ }^{3}$.

\section{Des moments road movie dans Kandahar}

Dans son article liminaire à ce numéro spécial de Cinémas, Walter Moser parcourt l'histoire du road movie américain et présente un certain nombre d'éléments qui lui sont constitutifs, 
tels que la combinaison cinéma-automobile, la route, la mobilité, l'arrachement à des espaces-temps fixes, la fugue, la re-nomadisation. Il circonscrit le noyau générique du road movie dans deux gestes essentiels qui sont: d'abord to hit the road, geste de libération qui implique rupture avec un ordre établi (le pouvoir paternel, par exemple) et signifie dégagement de la voie vers l'espace ouvert; ensuite to be on the road, geste de jouissance de la liberté dans l'espace ouvert qu'est la route. Comment Kandahar entre-t-il dans ce schéma propre au road movie américain?

"Un séjour au cœur de l'Afghanistan», tel est le sous-titre de Kandahar dont le scénario, intitulé Safar e Ghandehar, signifie d'ailleurs "Voyage à Kandahar». La notion de voyage "implique la route ${ }^{4}$, qui à son tour sous-entend l'espace ${ }^{5}$ ». Pour parcourir l'espace, il faut un moyen de déplacement. Dans Kandahar, le spectateur est invité à faire le voyage par différents modes de locomotion. La présence de moyens de déplacement dans les œuvres catégorisées sous le genre du road movie est un consensus minimal qui transcende les difficultés inhérentes à une délimitation claire des frontières du genre ${ }^{6}$. Considéré tantôt comme cinéma sur le sujet de la voiture ou autres moyens de locomotion (Corrigan 1991, p. 143-146), tantôt comme cinéma sur roue (Williams 1982, p. 6), le road movie est «à l'image des individus qu'il présente, [...] un vagabond qui erre dans le temps et les époques, présent d'un genre à un autre et sous différentes facettes ${ }^{7}$ ». La narratrice de Kandahar, Nafas, va effectivement errer, empruntant différents moyens de déplacement pour nous faire parcourir un espace diversifié. On commencera à la voir à bord d'un hélicoptère de la Croix Rouge survolant le territoire iranien. On voit dès les premières minutes du film qu'en lieu et place de la voiture personnelle, lieu commun du road movie américain, un moyen de locomotion plus rapide est mis au service de ce voyage. On apprend dans une conversation avec le pilote que Nafas prend l'hélicoptère parce que les autorités consulaires de l'Iran, du Pakistan et du Tadjikistan ont refusé de lui octroyer un visa. Sa fonction de journaliste dénonçant les conditions humiliantes des femmes du Moyen-Orient en général et de l'Afghanistan en particulier a fait d'elle une persona non grata. C'est donc pour éviter le passage 
des frontières terrestres qu'elle a recours, dans un premier temps, à l'espace aérien. Le désir de se rendre à Kandahar est plus puissant que toutes les barrières érigées sur sa route. Aussi mobiliset-elle tous les moyens disponibles pour arriver à destination. Elle est ensuite à bord d'un tricycle multicolore en compagnie d'une famille afghane. Cette famille fait avec elle un bout du chemin menant vers Kandahar avant de se faire voler des objets précieux et subtiliser le tricycle par les coupeurs de route. Plus tard, un autre road mate nommé Khak guidera Nafas, à pied, dans les dunes. Tahib Sahid, l'Américain noir, mettra à sa disposition son chariot, et Hâyat fera avec elle le reste du chemin, à pied.

Nafas monte à bord de l'hélicoptère non pas pour mieux contempler le sol en vue aérienne, mais pour contourner les murs terrestres représentés par le refus de visa. Si elle monte ensuite sur le chariot ou le tricycle, ce n'est pas non plus pour apprécier la splendeur des paysages. Ce sont les seuls moyens dont elle dispose pour se rendre à destination. Expérience pluriforme de la traversée des espaces qui donne envie de connecter Kandahar au cinéma de la route, au road movie. Le déplacement ainsi que les divers moyens de locomotion montrent que Nafas est on the road. Le chaînon manquant des deux gestes essentiels supposés constituer le noyau générique du road movie (Moser) est la déprise: to hit the road.

Être sur la route présuppose qu'on a pris une fois la route. Cela s'entend, mais la force du to hit the road réside dans la déprise, qui est un geste de rébellion caractérisé dans le Bildungsroman, le road novel et le road movie par le meurtre

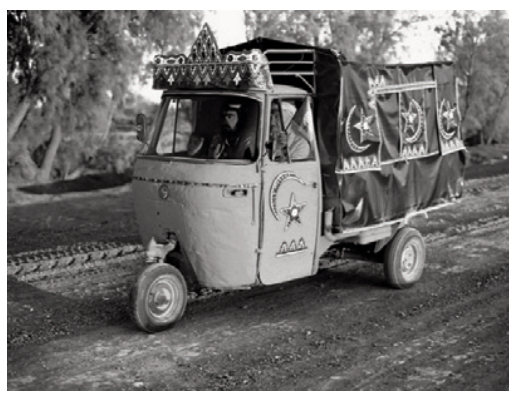

Tricycle pour Kandahar

(C) Seville Pictures symbolique du père ou la rupture avec la famille. Contrairement à cette notion de déprise, le départ de Nafas du Canada n'exprime aucune rupture. La déprise, si elle existe quelque part dans Kandahar, je la localiserai dans le grief de Nafas contre la burqa, une pratique religieuse propre à l'Afghanistan. Ce 
geste de Nafas contre la burqa peut être vu comme une rébellion contre une pratique qui a cours dans sa propre maison. Ainsi peut-on affirmer que dans Kandahar, la déprise a subi une modification majeure. Nafas prend la route non pas pour briser les amarres en fuyant cette pratique de voilement à laquelle elle s'est déjà soustraite. Elle ne prend pas le large pour respirer le vent de la liberté. Elle ne se dégage pas de ce qui retient, étouffe - topos essentiel du road movie classique. Au contraire, elle prend une route, dont la flèche directionnelle pointe vers Kandahar, pour aller affronter l'intégrisme taliban, donné dans le film comme dispositif contraignant ou barrière à fracasser. Tout se passe comme si l'on prenait la route pour aller à nouveau à la rencontre d'un adversaire que l'on a d'abord fui, et à qui l'on veut subtiliser des prisonniers restants. La quête de Nafas à la recherche de la sœur restée en Afghanistan suggère donc un projet ultime, qui verra cette sœur, symbolisant l'ensemble des prisonnières de la burqa, se déprendre du "rideau", de la cloison. Ainsi Kandahar présente-t-il dans sa structure, sous une forme modifiée à tout le moins, ce que Walter Moser appelle les unités minimales du road movie américain: prendre la route (to hit the road), être sur la route (to be on the road) et prendre la route à nouveau (to hit the road again). À cet égard, il est à considérer comme une variante du road movie.

\section{Des éléments d'interculturalité dans Kandahar}

En plus de participer au genre du road movie, le film tisse sa toile de fond sur un enjeu culturel. Déjà le titre Kandahar indique un lieu culturel précis vers lequel la route de Nafas est censée conduire. Le réalisateur est un Iranien ${ }^{8}$ qui tourne son film à la frontière irano-afghane et y fait jouer des Afghans, des Iraniens, un Américain noir, une Canado-afghane, des Européens travaillant pour la Croix-Rouge. En somme, des représentants d'un florilège de cultures. D'où l'intérêt de convoquer ici l'interculturalité. Que la notion d'interculturalité n'aille pas de soi est un constat fait par Pascal Gin dans son article liminaire. Pour conjurer les confusions conceptuelles et minimiser la charge sémantique de l'interculturel, Gin a essayé de baliser l'aire référentielle de l'interculturel dont il est question dans ce 
numéro, à savoir cohésion ou perturbation des identités culturelles, asymétries de la production culturelle, acquiescement critique à une opacité du culturel, etc. Je veux m'en tenir à cette aire référentielle en la reprécisant par rapport à l'objet filmique qui nous occupe.

L'hypothèse d'une constitution du film comme road movie interculturel se vérifie d'abord par la construction du personnage de Nafas qui se réclame de l'Afghanistan, terre de ses aïeux, et du Canada, terre d'accueil. Nafas est certes Afghane, mais elle est devenue Canadienne. En tant que Canado-afghane, elle représente une véritable charnière entre deux espaces culturels. Qu'est donc l'inter de l'interculturel si ce n'est pas, entre autre chose, ce trait d'union entre une culture $A$ et une culture $B$, trait représenté par ces personnes à double identité, culturellement hybrides, qui suivent ce qu'Irmela Schneider appelle la logique du Sowohl-als-auch, antipode de la logique monoculturelle Entweder-oder (Schneider 1997, p. 20). Par sa culture hybride, Nafas réalise dans son être le processus qui aboutit à ce que Fernando Ortiz a décrit sous le concept de transculture (Ortiz 1940), synthèse d'une perte culturelle (déculturation) et d'un gain culturel (néoculturation). Avant de se réfugier au Canada, Nafas a vécu sous le règne des Talibans. Elle a connu l'humiliation qui est le lot des femmes dans son pays natal régenté par la loi talibane qui taxe d'impure toute image, toute représentation visuelle. Au Canada, Nafas fait des expériences autres que celles qu'elle avait faites dans son pays natal. Elle redécouvre l'image sous un jour différent. L'image ne lui paraît plus impure. L'œil de la caméra se pose sur des réalités et les capte pour les conserver et les montrer. La représentation cinématographique sert ainsi de véhicule d'idées, de mémoire et d'archive. Or le cinéma se veut une alliance entre la technique photographique et « un système permettant d'enregistrer puis de restituer de façon satisfaisante l'analyse d'un mouvement réel " (Le petit Larousse illustré). Cette fonctionnalité de l'image que maîtrise Nafas, journaliste, elle va chercher à en faire profiter ses sœurs et frères afghans. Sa délicate position entre l'Afghanistan qu'elle a fui et le Canada qui est devenu sa maison fait d'elle le pion central du film, capable de déjouer ce qu'elle considère 
comme de l'intégrisme afghan, en jouant sur le code culturel qu'est le voile. D'ailleurs, le prénom afghan Nafas, explique le réalisateur Makhmalbaf, signifie «respiration». Si la burqa empêche «la femme de respirer et d'être libre» (Haghighat 2001), Nafas vient lui apporter respiration et liberté. Nafas, quête de la liberté, motif essentiel du road movie. Le nom est tout un projet. Nafas, dit le réalisateur, c'est «le symbole de la femme afghane, qui connaît une situation meilleure au Canada». Elle a fait l'expérience de l'altérité, expérience de l'ailleurs, et est supposée avoir un double regard par rapport à ses sœurs restées en Afghanistan. Elle peut, à l'occidentale, sortir de sa maison, parcourir le monde en hélicoptère, en tricycle à moteur, en chariot à traction animale, à pied... Elle peut, liant mobilité et émancipation, lever son voile et parler aux hommes en les regardant dans les yeux.

\section{Une route de dévoilement: hijâb versus kashf}

Mettre à l'écran ce qui empêche "la femme de respirer et d'être libre" est le projet que s'assigne le réalisateur pour, d'une part, révéler au monde les tribulations de la femme afghane et, d'autre part, apporter un peu de lumière à cette dernière à travers l'acte symbolique du dévoilement (lever son voile). Le hijâb (voile) désigne le linge qui couvre et évoque en Islam «la dissimulation des choses secrètes" (Chevalier 1977, p. 812813). Symboliquement, il s'oppose à kashf (dévoilement), qui "est une révélation». Le concept de "révélation" participe du débat hautement philosophique, je veux dire métaphysique, sur

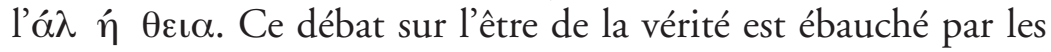
présocratiques Parménide et Héraclite, continué par les socratiques Platon et Aristote, repris par les modernes Descartes, Kant, Nietzsche, et relancé par le philosophe contemporain Martin Heidegger, qui y a consacré son œuvre la plus connue, Sein und Zeit (1927). Pour Heidegger (1965, p. 219), la définition d'Aristote qui fait de l'essence de la vérité l'accord du jugement avec son objet (adaequation intellectus et rei) est vide. L'essence de la vérité résiderait dans le dévoilement, Entdeckheit (Unverborgenheit) (Heidegger 1967, p. 219) plutôt que dans une adéquation simple entre un énoncé et l'objet sur lequel il 
porte. Pour expliciter cette équation vérité = dévoilement, Heidegger a dû recourir au mot utilisé par les anciens Grecs pour designer la vérité : $\alpha \lambda \hat{\eta} \theta \varepsilon\llcorner\alpha$. "La vérité, en tant qu'ó $\lambda$ ๆ $\theta \varepsilon\llcorner\alpha$, désigne ce qui a été soustrait (a-privatif) à l'oc-

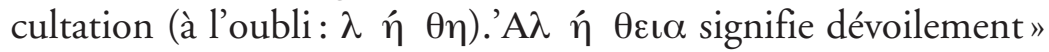
(Boutot 1989, p. 49). Ce concept traditionnel de la vérité présuppose, selon Heidegger, la non-vérité originaire, c'est-à-dire le mystère que la vérité révèle ou doit révéler. La vérité s'identifie donc, dans son essence, à la révélation, au dévoilement. "La vérité en tant que dé-voilement est intrinsèquement référée à la non-vérité, c'est-à-dire au voilement " (p. 49). Dévoiler, manifester, sortir de l'oubli, serait pour Heidegger l'essence de la vérité. Dans une conférence intitulée De l'essence de la vérité (Vom Wesen der Wahrheit, 1943), il complète son analyse et conclut avec le chiasme: l'essence de la vérité est la vérité de l'essence "das Wesen der Wabrheit ist die Wahrheit des Wesens" (Heidegger 1997, p. 29). Si l'essence de la vérité est le dévoilement, l'équation vérité $=$ dévoilement devient dévoilement = essence, qui est la nature profonde de l'être. L'être est alors "dans le fond, dévoilement, ouverture, éclosion dans la présence» (Boutot 1989, p. 52). Heidegger fait ainsi du dévoilement une question essentielle et existentielle. Le voilé, c'est le mystère appartenant potentiellement à l'horizon de la vérité dans la mesure où il se rend disponible au dévoilement.

Voiler et dévoiler renvoient donc à un geste naturel (mettre et enlever le voile) ainsi qu'à une réalité de l'ordre du mystère (dissimuler et révéler). Voiler, dévoiler: deux actes apparemment

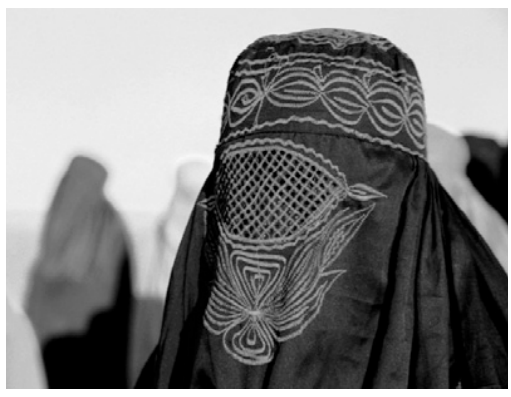

Nafas voilée

(C) Seville Pictures

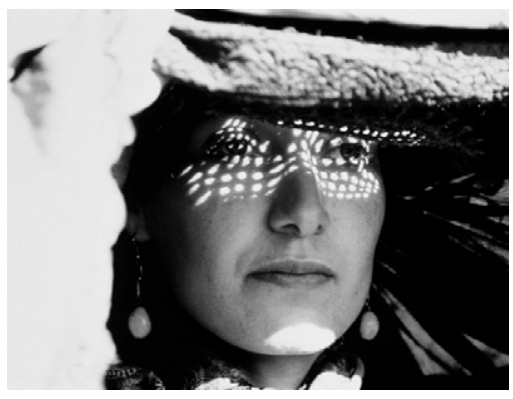

Nafas se dévoilant

C) Seville Pictures 
anodins. Deux actes contraires à des enjeux impératifs dans une pratique du politique, du social et du religieux que d'aucuns qualifient de fondamentaliste ${ }^{9}$. Dévoiler ce qui est voilé, révéler donc, un acte délicat qui évoque aussi, sur un plan épistémologique, l'Entdeckheit de Heidegger, c'est-à-dire la manifestation de l'identité. Pour comprendre comment, dans le film, se joue le jeu de voiler-dévoiler pour rendre manifeste ce qui est voilé, intéressons-nous aux gestes de la protagoniste Nafas. Ses gestes, ajoutés à certaines narrations intradiégétiques et à quelques propos extradiégétiques (interviews), révèlent le fond politicoreligieux du doublet voiler et dévoiler.

Les toutes premières images de Kandahar montrent Nafas au poste de sécurité irano-afghan. Un contrôleur l'interroge: "Comment t'appelles-tu?" "Nafas", répond-elle après avoir soulevé sa burqa. "Qui es-tu?" "La cousine de la mariée». Après quoi elle remet sa burqa. Cette discussion a en fait lieu dans la séquence finale du film. Mise en abyme, elle annonce les couleurs du voyage, au cours duquel la route devient un alibi, un théâtre pour représenter le combat entre le voilement et le dévoilement. À la fin du film, l'échange entre le contrôleur et Nafas est immédiatement suivi d'un commentaire de la jeune femme qui fait la lumière sur le projet de son voyage. "Je m'étais toujours échappée des prisons de femmes d'Afghanistan, mais aujourd'hui, je suis prisonnière de chacune de ces prisons, seulement pour toi, ma sœur." Qu'appelle-t-elle "prisons de femme", ici ? La principale, soit la burqa ${ }^{10}$, est définie hors caméra comme un "vêtement recouvrant entièrement la femme afghane» et l'empêchant «de respirer et d'être libre» (Haghighat 2001).

Lors de la première escale, pendant une séance de photos de famille, Nafas considère toutes ces femmes voilées et se demande si le port de la burqa relève de la culture afghane ou s'il s'agit d'une décision gouvernementale. Cette interrogation se mue aussitôt en dénonciation de l'écart entre le traitement réservé à la femme et celui qui est accordé à l'homme:

En Afghanistan, chaque ethnie a un nom et une image propres: Hazara, Ousbaks, Turkmènes, Tadjiks, Kirghiz, Nouristanis, Mongols, etc. Mais les femmes d'ici qui représentent la moitié de 
la société n'ont pas de nom ni d'image, car elles sont voilées. C'est peut-être pour ça qu’on les nomme "siya sar ", «têtes noires".

En laissant entendre que toutes les ethnies ont un nom, une image, alors que les femmes n'en ont point, Nafas oppose "femme» à "ethnie». Qu'elles soient de l'ethnie hazara, ousbak, turkmène, tadjik ou mongol, les femmes, contrairement aux hommes, n'ont qu'un nom, le même: "siya sar", "têtes noires". L'image de leur visage est cachée derrière la burqa. Voilà pourquoi Nafas se drape elle-même d'une burqa pour procéder au dévoilement, au sens propre comme au sens figuré. Son accoutrement lui permet d'une part de se découvrir en ôtant sa burqa ad libitum, et d'autre part de pénétrer, sans contrariété, l'espace géographique dont elle veut révéler les pratiques et mœurs jugées par elle non conformes aux normes de sa logique.

Pour dénoncer la cruauté des seigneurs de la guerre, le réalisateur du film aménage une escale à Niatak, village à la frontière iranienne. L'escale est un lieu commun du road movie, où l'arrêt à des stations d'essence pour faire le plein de carburant, pour prendre un café, fumer une cigarette ou simplement contempler le paysage devient presque un must. L'intéressant titre d'Anja Herkenrath qui semble induire que, dans le road movie, l'on ne "doit jamais s'arrêter» ("Niemals stillstehen” — Roadmovies " $"$ ) se révèle ici comme un trompe-l'œil. Nafas se plie à la règle des escales non pas pour des impératifs techniques — faire le plein d'essence, par exemple —, mais pour introduire le spectateur dans la vilenie de la guerre.

Lors de cette escale, Nafas assiste à la cérémonie du dernier jour d'école où les filles afghanes se préparent à retourner en Afghanistan. La monitrice, au milieu des enfants, leur explique comment éviter les mines antipersonnel. Elle leur montre de jolies poupées avec des habits multicolores. De très beaux jouets dans lesquels logent des mines. Ces poupées meurtrières avaient eu raison des jambes de la sœur de Nafas, comme le relate la voix-je:

Dans ce voyage, la guerre est partout. Les chiens se battent, les oiseaux se battent, les humains se battent. Un combat de coqs m'a rappelé le jour où, après la mort de maman, papa s'est enfui d'Afghanistan avec nous. Tu te souviens, ce jour-là, on a vu un 
combat de coqs. Les gens s'arrêtaient pour regarder. Papa et moi, on a été attirés par la foule mais toi, tu as vu une poupée dans le sable. Tu as couru vers elle, elle a explosé et tu as perdu tes jambes. Papa m’a confiée à une autre famille de réfugiés pour rester avec toi jusqu'à ta guérison. Mais tu n’as pas guéri. Papa est mort et tu es restée toute seule.

Faute d'effet flash-back — fondu enchaîné facilitant le glissement du présent au passé (Vernet 1980, p. 96) —, le réalisateur se sert de cet élément audio pour nous livrer une petite partie de la vie antérieure de Nafas en Afghanistan. Plus encore, il s'agit de nous renseigner sur le sort qu'a été celui de cette sœur symbolique que Nafas tente de sauver par tous les moyens, le sort finalement de ces enfants innocents dont la vie est à jamais brisée par des poupées-mines. Dans le film, la poupée n'est plus un jouet inoffensif, mais un engin de guerre. Ces mines camouflées ont causé la perte des jambes de nombreux Afghans. La caméra, depuis l'hélicoptère, capte le mouvement de ces hommes, femmes et enfants qui se bousculent pour des prothèses parachutées par la Croix Rouge. Des jambes pour marcher, voilà l'objet de la convoitise de ces estropiés. Les images de cette plongée mettent en branle les fibres émotives du spectateur.

Une autre escale nous amène dans une école coranique. Le film donne à voir, dans une salle de classe, l'intrusion dans le sacré d'un ordre de violence. Le mollah fait réciter le Coran et interroge quelques enfants sur le fonctionnement des armes:

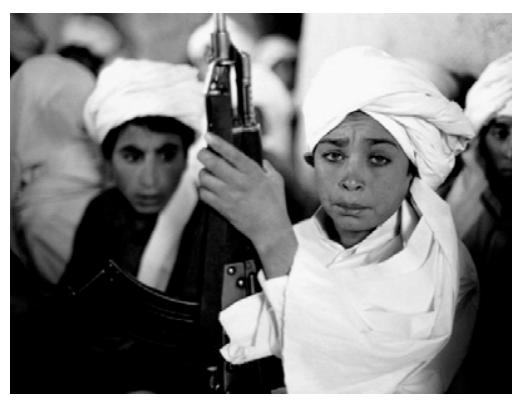

À l'école coranique

(C) Seville Pictures

— Qu'est-ce qu'un sabre?

- Une arme qui exécute l'ordre de Dieu (Allah). Elle coupe la main du voleur et la nuque de l'assassin.

- C'est quoi la Kalachnikov?

- Une arme semi-automatique et mécanique avec poudre propulsive et ressort. Il tue les vivants et défigure tout corps déjà mort. 
La clarté du son et la rapidité du rythme dans cette scène révèlent que les enfants sont parfaitement initiés aux idées guerrières en lieu et place de la religion. L'agencement des images de ces étudiants-guerriers donne lieu de croire qu'il y a, de la part du réalisateur, une volonté manifeste de mettre au pilori l'instrumentalisation des enfants sous le prétexte de la religion. Les enfants ne sont plus des naïfs, mais des guerriers potentiels qui doivent lutter pour survivre. Cette métaphore du meurtre de l'enfance que propage le film a une fonction de dévoilement, c'est-à-dire de dénonciation des conditions de misère dont sont spécialement victimes les femmes et les enfants. Et la narration filmique le dit: "Un jour, le monde verra votre situation et vous aidera."

La situation dont il est question, le parcours de Khak constitue un cas exemplaire qui la met en relief. On le voit pour la première fois parmi les élèves de l'école coranique. Tandis que ses deux camarades récitent avec brio leur leçon sur les armes de guerre, Khak éprouve quelques difficultés à réciter son Coran. Il est renvoyé de l'école pour motif d'incapacité. Il lui faut se débrouiller pour trouver sa propre pitance et celle de sa mère, son père étant mort pendant la guerre. Nous le trouvons plus tard errant de cimetière en cimetière et récitant, à sa façon, le Coran pour gagner quelques sous. C'est dans l'un de ces cimetières que sa route croise celle de Nafas. Il accepte alors, moyennant une récompense pécuniaire, de lui servir de guide sur le chemin de Kandahar. Jusque-là, le film nous a dévoilé la débrouillardise plutôt louable de Khak. Mais en suivant la marche de Nafas et de son guide, après quelques kilomètres, on comprend l'enjeu que représente Khak dans le projet global du film. En effet, leur route bifurque. Khak propose de prendre la route la plus longue. Or, ce dont Nafas dispose le moins, c'est de temps. Elle a devant elle trop d'espace à parcourir pour rallonger inutilement le parcours. Malgré l'air désemparé de Nafas, Khak insiste. Selon lui, plus longue sera la route, plus il s'y trouvera des objets à prendre et à revendre. L'objectif de Khak devient alors plus clair: se servir de la route pour recycler des objets kitsch ou des bricoles et les revendre pour quelques sous. Devant l'insistance de Nafas à prendre le plus court 
chemin, Khak n'hésite pas, il l'abandonne. Il prend sa longue route. La caméra suit Nafas. Très vite, elle rebrousse chemin et part à la recherche de son jeune guide. La caméra continue à la suivre, puis un zoom nous entraîne, en même temps que Nafas, vers une découverte macabre: Khak accroupi sur un cadavre en décomposition dont il fouille les restes. Il y déniche une bague qu'il brandit en direction de Nafas qui, aussitôt, s'enfuit. Khak la poursuit avec la bague du cadavre pour la lui vendre. En tout cas, il obtiendra quelques dollars contre ce bijou, comme ce fut le cas pour une chanson. L'adversité a appris à Khak à monnayer tout ce qui lui tombe sous la main. En cet enfant, vrai picaro contemporain tatoué par la misère, la naïveté cède à la lutte pour la survie ${ }^{12}$.

Après l'école coranique et la traversée du cimetière avec Khak, la route de Nafas nous conduit chez Tabib Sahid, le médecin de village. La mise en scène de cette escale pour consulter le médecin est l'occasion de montrer une pratique de la société afghane: la barrière érigée entre l'homme et la femme, afin que cette dernière demeure cachée aux regards des hommes. La matérialisation de la barrière se fait non plus avec le voile - puisque la patiente se dévoile en consultation -, mais par une barrière physique érigée comme un confessionnal entre le médecin et sa patiente, ne laissant qu'un trou suffisamment grand pour que le médecin puisse voir la bouche ouverte, l'œil ou l'oreille de sa patiente. Dans ce "confessionnal", la communication entre médecin et patiente n'est pas directe, elle passe par la médiation des enfantstraducteurs. Si le «transfert culturel est une sorte de traduction puisqu'il correspond au passage d'un code à un nouveau code" (Espagne 1999, p. 8), l'épisode de la consultation montre un transfert de code qui ressemble bien à une traduction, mais à une traduction sans changement de code. Lors d'une première consultation, on voit une petite fille nommée Nafasgul entre la patiente, sa mère et le

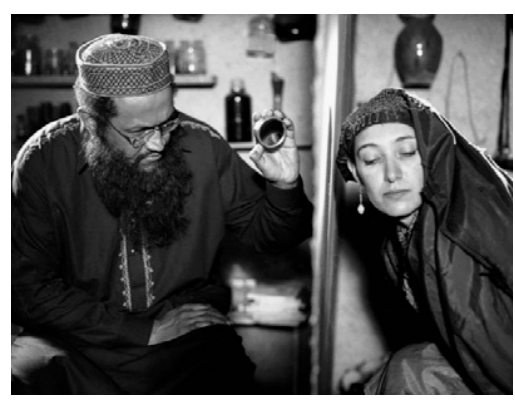

Au cabinet du médecin (C) Seville Pictures 
médecin. Le rôle de l'enfant: retransmettre fidèlement à sa mère ce que dit le médecin, et vice versa. Deuxième consultation: Nafas arrive avec son guide Khak. Elle a, en cours de route, bu l'eau du puits du village et a eu la diarrhée. Comme Nafasgul dans la consultation précédente, Khak occupe la place située entre la patiente Nafas et le médecin et joue le même rôle que la petite fille.

Les enfants-interprètes ont, du point de vue de la traduction, une valeur zéro, car ils n'ajoutent ni ne retranchent rien à ce que dit le médecin; ils ne traduisent pas non plus dans une autre langue. En se contentant de répéter fidèlement les paroles du médecin, ils jouent néanmoins un rôle important dans le mécanisme du voiler-dévoiler, un rôle important pour l'entretien d'une pratique culturelle. Le médecin homme n'est pas autorisé à avoir un contact direct avec sa patiente qui se dévoile. La présence des enfants sert à conserver le voile de la femme dévoilée. Kandahar illustre ainsi une des fonctions ${ }^{13}$ que Nadine Weibel attribue au voile, à savoir la fonction de séparation. Le voile, écrit Weibel (2000, p. 76), «sépare en cachant, protège ce qu'il cache d'éventuelles influences pernicieuses. Avant toute chose il sépare les femmes des hommes".

Lévolution concomitante de ces deux contraires que sont voiler et dévoiler est permanente dans le film. On peut y voir une allégorisation du rôle que Nafas, en tant que journaliste, joue pour lever le voile sur la situation d'inégalité que vivent les femmes afghanes. D'ailleurs, l'objectif du voyage qui consiste à sauver la sœur participe de cette allégorisation. Montrer au monde la situation des femmes afghanes, voilées sous la burqa, confinées entre les quatre murs de leur maison, semble être l'un des projets de Kandahar. Le voile, concrètement barrière au regard visuel de l'homme, devient métaphoriquement le symbole de ce mur imaginaire qui sépare les femmes afghanes du monde extérieur.

\section{Le dévoilement comme subversion}

Prendre la route dans le road movie s'accompagne toujours, sinon presque toujours, du désir de rompre avec, de braver ou de renverser l'ordre établi. Mais peut-on changer un ordre sociale- 
ment établi, des idées et valeurs reçues et intériorisées sans courir un certain risque? La route de l'émancipation n'est pas sans embûches. Aussi l'attitude émancipatrice de Nafas vis-à-vis des hommes lors du tournage de Kandahar sera-t-elle vilipendée par une femme afghane, comme le rapporte Pazira (2001) dans le commentaire intégré extradiégétiquement au film: "Un jour, une vieille femme m'a crié devant tout un groupe d'hommes silencieux: "Retourne chez toi, femme impudique. N'as-tu pas d'honneur pour parler aux hommes de cette façon et travailler avec eux ainsi ?" "L'intervention de cette femme qui semble bien s'accommoder de sa situation de voilée, de sa vie derrière la burqa, nous introduit dans une logique opposée à celle représentée jusqu'ici par Nafas. Contrairement à la logique de Nafas qui prône le dévoilement du mystère (du voilé) pour actualiser sa valeur intrinsèque, la logique de la vieille femme pose la femme comme un mystère qui doit demeurer caché, voilé. Dans cette logique, la femme a une valeur certaine lorsqu'elle est voilée. Dévoilée devant un public masculin, elle devient "impudique", c'est-à-dire indécente, contraire à ce qui convient. Ce qui convient dans la logique de la vieille dame, c'est la sauvegarde du mystère, le voilement, la vie derrière la burqa, la vie sans image ${ }^{14}$. Il ne s'agit pas de l'image en soi "présente sans être représentée " (Liandrat-Guigues 2005, p. 24) dont parle Henri Bergson dans Matière et mémoire, mais d'image au sens de représentation, d'exposition à la vue, de présentation au regard. En somme, image au sens de soustrait à l'occultation. Occulter, c'est dissimuler, rendre moins apparent. Ce qui est apparent est visible, manifeste, c'est-à-dire évident et reconnu de tous. Vivre voilé, c'est finalement vivre méconnu de tous. Pazira arrive avec une équipe d'hommes, munie de caméras et d'un arsenal cinématographique, pour soustraire à l'occultation ce qui est dissimulé au regard, pour arracher de l'oubli ce qui est voilé. Elle est venue voilée pour faire

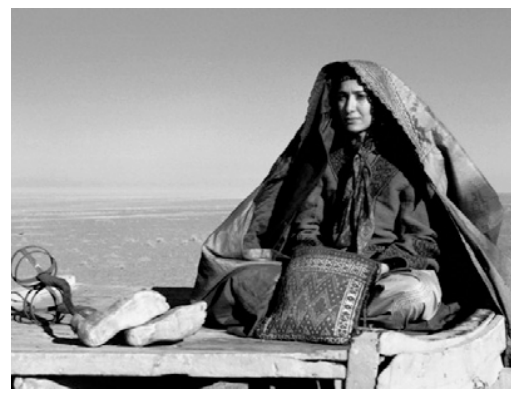

Nafas dévoilée

(C) Seville Pictures 
tomber le voile, pour révéler au monde (entdecken) ce qui est voilé (das Gedeckte). Le malaise de la vieille dame devant l'attitude de Pazira, qui vient ainsi bousculer la tradition en introduisant la représentation d'images dans cette sphère antiimage, peut s'expliquer par sa croyance tenace aux pratiques religieuses et ancestrales. Du point de vue du réalisateur, l'altercation entre cette vieille femme, respectueuse de la tradition, et Pazira n'est pas à loger au compte du choc des cultures. Il s'agit d'une guerre de la culture contre l'inculture, une guerre contre le désespoir. Et le film le rend bien par l'entremise de la voix narratrice:

\footnotetext{
J'ai donné mon âme à ce voyage, j'ai pris des chemins inexplorés pour te donner une raison de vivre. J'ai traversé le champ de pavot et j'ai rencontré des inconnus pour puiser de l'espoir pour toi dans leurs rêves. Aujourd'hui, je t'amène mille magnifiques raisons de vivre.
}

En effet, le film donne à voir les multiples facettes du désespoir. Un regard rétrospectif sur le parcours de Nafas permet de revoir cette route sur laquelle Hâyat a dû extorquer des jambes artificielles en inventant toutes sortes d'histoires pour les marchander afin de survivre; cette route sur laquelle les enfants sont obligés d'avoir une place à l'école pour pouvoir manger ; cette route sur laquelle Khak est contraint de fouiller les restes d'un cadavre, de voler une bague et de la revendre. Un regard jeté sur la route de Nafas laisse voir qu'il y a bien des éléments dans ce film pour dire la misère lancinante du peuple afghan. Ainsi, bravant un des lieux communs du road movie, le réalisateur met sur la route une femme ${ }^{15}$ pour explorer et narrer la misère de sa société. Il se convainc qu'il ne s'agit pas seulement d'une misère matérielle dont souffrirait le corps, mais aussi d'une misère culturelle, une misère devant laquelle une femme peut s'émouvoir et émouvoir les spectateurs. Pour lui, les bourreaux des Afghans, ce sont les Talibans, ces mollahs qui considèrent impur tout ce qui est du domaine de la représentation: image, son, éducation. Le livre, l'instrument de musique et le magnétophone confisqués dans la scène du poste de contrôle symbolisent ces interdictions. Pas de cinéma, pas de télévision, pas de musique. 
Aussi le tournage du film dans le fief des Talibans prend-il l'allure d'une provocation frontale, car il se fait critique des Talibans qui ont dit non à toute forme de représentation par l'image et le son. Le cinéma comme production d'images mouvantes et de son se pose ici en refus du refus d'image. Dans le même ordre d'idées, la mise en scène du voyage fonctionne ici comme un refus du cantonnement dans l'espace, le réduit des villes sous le contrôle des Talibans. Le réduit du voile, antithèse de l'espace ouvert qu'est la route. Le voilement, caractéristique de la restriction des plaisirs visuels, devient l'élément sur lequel joue le réalisateur pour mener à bout sa provocation. Voilement (étouffoir) vs dévoilement (liberté). Binarité certes moraliste, mais pleinement assumée par la narration filmique. Plusieurs fois on verra Nafas enlever sa burqa dans le film. Il faut qu'elle montre son visage, qu'elle sorte de cet étouffoir qu'elle ne porte d'ailleurs que pour mieux l'enlever sous le feu des projecteurs.

Comme si autant de provocation ne suffisait pas, elle refuse de se séparer de son magnétophone, même en présence des contrôleurs talibans. La présence de ce petit appareil a une signification particulière, car ce dispositif technique permet l'enregistrement et l'archivage de certains moments clés du voyage. Nafas l'appelle "boîte noire», car l'appareil lui permet de transférer ce qu'elle dit ou entend sur bande sonore conservable. Comme dans un crash d'avion, la boîte noire est un témoin, celui qu'on interroge pour lire le déroulement du voyage, pour en tracer la carte. Elle est une mémoire du voyage, mais elle représente aussi la dernière source d'espoir que Nafas veut apporter à sa sœur. S'en séparer, c'est renoncer à l'essence même de son voyage, qui est avant tout témoignage. L'enregistrement, c'est la possibilité de témoigner. Plus de magnétophone signifie plus d'archives, plus de souvenirs. Le magnétophone allégorise ainsi la définition que Bédard (1990) donnait du cinéma, à savoir un dispositif technologique d'archivage et de saisie par l'image de l'histoire du monde.

La décision de tourner en Afghanistan est un acte qui consiste - j'emprunte ici les mots de Sharon Willis (Cohan et Hark 1997, p. 290) - à introduire le "déviant" dans le «normal». Dans la vision des Talibans, le cinéma est un déviant. 
"Au moment où Mohsen Makhmalbaf commençait à tourner Kandahar, ceux qui allaient en être les vedettes n'avaient jamais entendu parler de cinéma» (Pazira 2001). À en croire Pazira, dans l'Afghanistan des Talibans, non seulement l'image était perçue comme quelque chose d'impur, mais toute l'industrie du cinéma ployait sous le joug d'une censure implacable, résultat d'une interprétation talibane de la Parole révélée. La normalité talibane, c'est l'absence d'images, c'est la femme cachée sous la burqa. La discussion entre Nafas et l'homme dont elle prétend être la quatrième femme est éloquente à ce sujet: l'homme tient à ce que Nafas porte le voile si elle veut se faire passer pour sa femme. "C'est une question d'honneur. Nous sommes religieux. Aucun homme ne doit voir ma femme. Les gens se moqueront de nous si ta burqa n'est pas mise correctement. La burqa n'est pas un ornement. C'est un voile pour couvrir. " C'est donc pour le monsieur une question d'honneur que de veiller à ce que toutes ses femmes soient complètement couvertes. Et ce respect serait légitimé par la religion. Le port du voile serait en effet une prescription dans les textes du Saint Coran et les Hadiths de Mahomet, l'envoyé d'Allah: "Prophète, prescris à tes épouses, à tes filles et aux femmes des croyants d'abaisser un voile sur leur visage. Il sera la marque de leur vertu et un frein contre les propos des hommes. Dieu est indulgent et miséricordieux" (Sourate XXXIII, v. 59). Le port du voile est donné pour marque de vertu et de pudeur. Comme impératif moral ancré dans le sur-moi collectif, il est perçu comme l'habillement féminin normal pour paraître immaculée devant Allah sans exciter l'appétit insatiable des hommes. Le «normal» chez cet Afghan moyen, c'est voiler le visage des femmes pour être en paix avec sa société et en accord avec sa conscience. Le réalisateur introduit la subversion ${ }^{16}$ dans ce milieu qu'il explore avec sa caméra. La caméra sur la route vers Kandahar prend ainsi en charge le combat pour l'émancipation de la femme musulmane (Saadaoui 1982), combat qui dénonce l'instrumentalisation du religieux ${ }^{17}$. Cette subversion s'exprime dans la construction même du rôle de Nafas qui, par le voilement et le dévoilement, vient bousculer des tabous intériorisés. Et c'est sa situation entre deux cultures qui lui permet d'être aussi audacieuse pour braver les tabous. 


\section{Des incongruités du projet interculturel}

Grâce à la bravoure et la prestation de Nafas dans Kandahar, le film a eu un bon accueil auprès du public (occidental surtout). Cet accueil est redevable aussi bien au sujet traité qu'à l'approche du réalisateur ${ }^{18}$, une approche pragmatique qui met l'accent sur la démonstration à faire. "Dans ce genre d'approche, le risque est grand que celui que l'on filme devienne moins important que la démonstration qu'il permet de faire, et qu'il demeure de ce fait dans son étrangeté radicale»(Loiselle 2005, p. 9). Kandahar a-t-il été à l'abri de ce travers? Rien n'est moins sûr.

L'amalgame entre arme de guerre et enseignement coranique induit un glissement du bellicisme vers le terrain religieux et vice versa. Ce glissement a pu être interprété comme une défiguration de l'islam qui serait assimilé ici à la violence. La débrouillardise de Khak donne de lui l'image d'un enfant prêt à tout, même à déterrer les cadavres pour avoir de l'argent. Si Nafas est le symbole de la respiration, ne peut-on pas voir dans son périple à la recherche de sa sœur une métaphore de la femme-lumière à la rescousse de ses sœurs qui baignent encore dans les ténèbres sans nécessairement le savoir? Sa situation entre deux cultures la mettrait-elle un peu au-dessus des autres femmes afghanes? L'objectif général de dévoilement (révélation) que s'assigne Kandahar n'est-il pas assimilable à la prétention du cinéma documentaire qui fait dire à Hitchcock ironiquement que "Dieu est l'auteur du documentaire» (Gaudreault et Marion 1994, p. 12)? Ce n'est cependant pas pour ces raisons que le film, salué par de nombreux prix et acclamé par plusieurs journaux, est controversé.

Au centre des controverses se trouve le personnage de Tahib Sahid dans le rôle du médecin de village. Il entre en scène à la dixième séquence intitulée Un étranger amical. Cette séquence met en scène une multiplicité culturelle: Sahid, un Américain noir, Khak, un Afghan, Nafas, une Canado-afghane... Venu combattre dans l'armée russe, l'Américain noir, converti en chercheur de Dieu, est un personnage sympathique. Le soldat tueur d'hommes a troqué sa Kalachnikov contre un bistouri pour se transmuer en soigneur d'hommes depuis qu'il a rencontré, dans la misère absolue, deux petits enfants pachtoune et 
tadjik, deux ethnies qu'il a tour à tour combattues. C'est dans le service aux soins des hommes qu'il espère rencontrer le Dieu qu'il cherche. Une femme afghane, visiblement dans le besoin, vient voir le médecin, non pas pour consulter, mais pour lui vendre des poules. S'étant rendu compte que les poules sont malades, il lui donne de l'argent et lui conseille de ne pas vendre ni manger les poules. Lors de la consultation de Nafas, l'Américain prend la liberté d'exclure l'enfant-traducteur Khak pour discuter directement avec la patiente en anglais. Une exclusion masquée par quelques questions sporadiques lancées à Khak pour lui donner l'impression qu'il est toujours dans son rôle de traducteur. En tête-à-tête avec Nafas, l'Américain se renseigne sur l'identité du traducteur :

— Qui est ce garçon? Tu le connais ? [...]

- Je ne le connais pas. C'est mon guide. Je l'ai rencontré dans un cimetière. C'est le seul qui ait accepté de m'amener à Kandahar.

— C'est dangereux. [...] Il n'est pas fiable. Laisse-le partir. La misère fait faire n'importe quoi.

Le propos selon lequel Khak ne serait pas fiable frise le mépris de l'autre et suggère à Nafas de se méfier de l'Afghan Khak pour se fier plutôt à l'Américain Sahid, ce qu'elle fera d'ailleurs en se séparant de Khak pour confier son sort à Sahid. Celui-ci, dans la scène, est donné pour celui qui comprend bien le problème de Nafas et cherche à l'aider au mieux. Il tiendra sa promesse de faire avec elle un bout du chemin.

Sur la route, dans le chariot de Sahid, les voyageurs rencontrent un paysan du coin que l'Américain, malgré la méfiance redevable à la situation de guerre, accepte de conduire au centre de la Croix Rouge. Au centre, ils apprennent qu'un certain Hâyat est en route vers Kandahar. Ils se précipitent pour le joindre et lui demander d'y aller avec Nafas. Mais Hâyat est plus préoccupé par le fait de gagner sa vie que de se rendre à Kandahar. Il profite donc de la présence des étrangers pour marchander la paire de jambes artificielles qu'il vient de prendre à la Croix Rouge, où, en vrai mythomane, il a dû inventer histoires sur histoires pour se faire remettre des prothèses, lui qui a encore ses deux jambes. 
Avant d'accepter de servir de guide à Nafas, il fait monter les enchères, présentant l'image d'une personne qui vendrait son âme au diable pour quelques sous. Alors que Hâyat, tout comme Khak, s'efforce de vendre ses services, l'Américain reste près de Nafas avec qui il discute de temps à autre en anglais, ce qui exclut évidemment Hâyat, comme ce fut le cas de Khak. Une complicité se développe rapidement entre l'Américain et la Canado-Afghane avec pour conséquence l'exclusion — sporadique à tout le moins - des Afghans qui inspireraient peu confiance. Alors que l'Afghan est peint sous son mauvais jour, l'Américain joue le rôle d'étranger amical. Enlevant sa fausse barbe, il dira à Nafas: «Moi aussi, je dois sortir de derrière le rideau. C'est une burqa pour homme, de la même façon que ce que tu portes est une barbe pour femme. [...] Peut-être un jour ils comprendront qu'il faut vraiment enlever ces rideaux.»

Sahid marque ainsi un changement dans le registre de la dénonciation. Ce n'est plus seulement les murs des femmes mais aussi ceux des hommes qui sont décriés, et Sahid est le porteparole de cette élévation à l'échelle universelle du dévoilement qui s'accomplit avec le déplacement de Nafas. Ainsi, la rencontre des diverses cultures est mise à mal par ce déséquilibre ${ }^{19}$ dans les rôles assignés selon qu'on vient de l'Amérique du Nord ou du Moyen-Orient. La posture de Sahid vis-à-vis de l'altérité renvoie beaucoup plus à une volonté d'inférioriser l'autre (Afghan), sinon à tout le moins de l'assimiler à une certaine conception occidentale (nord-américaine) qui associe le voile à la prison de la femme. Aussi la scène de rencontre culturelle reproduit-elle le schéma d'une acculturation plutôt qu'une rencontre culturelle féconde. En disant: "Moi aussi, je dois sortir de derrière le rideau ", Sahid évolue dans une logique du rejet de la barbe et de la burqa. Ces dernières sont pour les Afghans, en tout cas pour les Talibans, des éléments religieux et culturels que Sahid assimile tout simplement au rideau (entendre cloison, écran qui cache). Dans ces propos cités ainsi que dans l'attitude générale de Sahid, on ne voit aucun signe qui montre une certaine attente de sa part par rapport à la culture afghane, la culture de l'altérité. Ce développement nous ramène à notre hypothèse de départ, à savoir si l'étiquette de film interculturel convient à Kandahar. Si 
l'interculturalité s'énonce comme une coprésence de cultures qui s'enrichissent mutuellement, l'une et l'autre acceptant les pertes compensées par les apports utiles de l'alterculture, on aura bien du mal à classer Kandahar comme un film interculturel. Seulement ce rapport fécond entre cultures est plutôt la transculturation à la Ortiz. Kandahar est un film qui met en présence plusieurs types de rencontres culturelles. À ce titre, on pourra le lire comme un film multiculturel ${ }^{20}$, mais je le traite comme un document interculturel, en ce sens qu'il permet de problématiser l'interculturalité, énoncée ici comme cohésion mais aussi comme perturbation des identités culturelles.

\section{Conclusion}

Il s'est agi dans cet article d'analyser le film Kandahar sous un triple angle: celui de son appartenance au genre cinématographique du road movie ; celui de l'interculturalité qui est ici manifeste dans le hasard des rencontres culturelles et surtout dans la double identité de Nafas; et enfin, celui théologicophilosophique avec la question de voile/dévoilement et les rapports humains entre sexes qui en découlent. En fin de parcours, on peut constater que la narration de la route dans Kandahar sert d'expédient pour promener le spectateur dans les méandres de l'Afghanistan des Talibans. Contrairement au road movie américain, qui reproduit le mythe de l'individualisme (Cohan et Hark 1997, p. 3), la route de Nafas nous oriente vers les clivages entre hommes et femmes afghans. La narration de la route révèle la misère orchestrée par des forces politico-religieuses. La route devient le lieu privilégié de révélation des dysfonctionnements sociaux. Plusieurs éléments incongrus de dévoilement décelés sur la route permettent de s'interroger sur l'attitude à adopter devant la misère lancinante de l'autre. La dénoncer ou y rester indifférent? Si l'on n'est pas indifférent, quel regard poser sur l'autre pour que sa misère ne devienne pas un alibi pour faire du sensationnel ou pour dénigrer l'altérité?

Nafas a, en tout cas, osé faire le pas de l'intrusion dans l'espace de l'autre. Son déplacement s'inscrit dans les éléments constitutifs du genre du road movie où le protagoniste se libère des cages sédentarisantes, avec la nuance ici que Nafas ne se met 
pas en route pour sa propre libération, mais pour libérer les autres. Et son itinéraire prend fin au poste de sécurité, à une frontière imaginaire que le spectateur ne peut identifier à Kandahar. Entrera-t-elle dans Kandahar? Sauvera-t-elle sa sœur? Nemo sciit. Le voyage s'interrompt. La même éclipse solaire qui a ouvert le film le termine. Le soleil disparaît sous l'ombre de la lune, indiquant peut-être que la lumière de Nafas n'arrivera pas au destinataire. Une fin au point de départ.

\section{Université d'Ottawa}

\section{NOTES}

1. Nalofer Pazira est d'origine afghane. À l'âge de seize ans, elle a fui la guerre en Afghanistan pour se réfugier au Canada, pays dont elle a obtenu la citoyenneté. Elle y a fait des études journalistiques et a exercé comme journaliste. Elle joue dans Kandahar le rôle de Nafas, narratrice à la voix-je (Vanoye et Goliot-Lété 1992, p. 36), car il s'agit en fait d'une mise en scène de sa propre vie. Propulsée dans le rôle du personnage principal, elle est au centre de la fiction et "propose la lecture de l'image et du spectacle filmique» (Vernet 1980a, p. 178).

2. Le voile est une toile légère qui couvre sans cacher complètement pour susciter l'envie de découvrir ce qu'il cache. Il est supposé entretenir un mystère qu'on a envie de découvrir. Or, la burqa cache complètement le visage. C'est un cas limite de voile.

3. Le cadre théorique étant élaboré dans l'introduction à ce numéro spécial de la revue Cinémas sur "Le road movie interculturel" par Walter Moser, en ce qui concerne la constitution générique du road movie (voir "Le road movie: un genre issu d'une constellation moderne de locomotion et de médiamotion»), et par Pascal Gin quant aux constituants interculturels du genre (voir «Les bifurcations culturelles du road movie contemporain»), je m’y référerai en le précisant pour le cas qui nous concerne.

4. Le mot français route partage avec son équivalent anglais road le sens de "voie carrossable, aménagée hors agglomération " pour les automobilistes, mais il évoque également le "trajet d'un avion ou d'un navire», en somme l'espace à parcourir ou l'«itinéraire à suivre pour aller d'un endroit à un autre» (Le petit Larousse illustré).

5. Stéphane Benaïm, "De l'errance au road movie dans l'œuvre de Jim Jarmusch", Mémoire de DEA de cinéma disponible en ligne à l'adresse suivante: < http ://www. kino-road.com/articles.php >.

6. Voir Santiago García Ochoa, "I Drive Therefore I Am: Norteamérica y el automóvil ", disponible en ligne à l'adresse suivante: < www.ucm.es/info/especulo/ numero30/idrive.html >.

7. Stéphane Benaïm, op. cit.

8. En tant qu'Iranien, le réalisateur agit en position de sujet observant l'altérité culturelle à Kandahar. L'asymétrie de regard entre le sujet observant et l'objet observé n'est pas totalement absente du film. Je relèverai ultérieurement ces éléments incongrus qui nous montrent ce regard condescendant sur l'altérité. Pour l'instant, je 
continue l'analyse des rencontres culturelles à partir de la position du réalisateur en m'appuyant sur les dialogues, les soliloques tirés du film ou encore sur des interviews accordées par le réalisateur et Pazira (Nafas). Ce positionnement permettra de minimiser les risques de la subjectivé inhérente à l'exercice d'interprétation.

9. Voir Femmes voilées. Intégrismes démasqués (Geadah 1996). On consultera aussi à ce sujet Abdelaziz Kacem (2004, p. 48) pour qui le voile «est une affaire essentiellement politique", un enjeu de pouvoir entre intégrisme chrétien qui cherche à barrer la route à l'intégration des minorités musulmanes et l'islamisme qui profite de cet ostracisme pour récupérer du terrain. Le voile, enjeu de pouvoir entre deux extrémismes qui se nourrissent l'un et l'autre, mais aussi enjeu politico-financier. Kacem signale en effet les propagandes télévisuelles en faveur du voile généreusement sponsorisées par al-Mutahajjiba (= la femme voilée), une entreprise spécialisée dans la confection et la commercialisation du voile. Et il conclut: "Au propre et au figuré, le voile constitue un fonds de commerce véritable» (p. 60).

10. Un voile est en principe assez léger pour permettre de respirer, de voir à travers et peut-être même de deviner ce qu'il cache. La burqa, telle que montrée dans Kandahar, ne répond à aucune de ces caractéristiques.

11. Disponible en ligne à l'adresse suivante: < http://www.xn--ldtke-kva.org/reisen/ roadmovie.htm >.

12, Une survie qui paradoxalement met la vie de Khak en péril. Il est en effet la seule personne à accepter de faire cette portion de la route avec Nafas. Tous les adultes ont décliné l'offre, sachant que la route est partout semée de mines antipersonnel.

13. Nadine B. Weibel (2000, p. 75-84) attribue cinq fonctions au voile: 1) une fonction d'attestation de soumission. Le voile signe la soumission inconditionnelle de la femme croyante à Dieu ; 2) une fonction de séparation. Le voile sépare pour protéger des possibles poussées de désir; 3) une fonction d'identification. Le voile marque la spécificité sexuelle, religieuse et culturelle; 4) une fonction de contestation. Le voile est signe du refus de l'assimilation en Occident; 5) une fonction d'instrumentalisation. Le voile est utilisé par les adolescentes soit pour tranquilliser les parents et échapper à une stricte surveillance, soit à des fins matrimoniales, partant du présupposé que les hommes s'amusent avec les filles «modernes» et épousent celles de «bonne moralité».

14. Plusieurs critiques du film Kandahar ont fait allusion à cet enjeu d'images. Je donne deux titres éloquents à ce sujet: "Kandahar ou les premières images d'un pays sans images" (Levieux 2001, p. 43) et "The Land Without Face» (Macnab 2001, p. 7).

15. La femme sur la route est un phénomène plutôt récent qui a fait l'objet de quelques études, dont celle d'Amelie Soyka, Raum und Geschlecht. Frauen im Road Movie der 90er Jahre. On notera que Thelma and Louise (1991) de Ridley Scott est l'un des premiers road movies où des femmes tiennent les rôles d'héroïnes.

16. Parlant des offensives cinématographiques contre les tabous sexuels et le puritanisme, Amos Vogel (1977) considère le cinéma comme un art subversif.

17. Juliette Minces fustige l'instrumentalisation de la religion pour maintenir la femme sous la domination de l'ordre phallocratique. Certaines prescriptions des livres saints, pense-t-elle, refléteraient "la réalité des rapports sociaux existant à l'époque de la Révélation" (Minces 990, p. 229). Dans le même ordre, Abdelaziz Kacem (2004, p. 55) estime que les deux sourates qui font allusion au voile (XXIV, v. 31 et XXXIII, v. 59) répondent à une "situation donnée, un événement particulier», d'où leur portée plutôt "transitoire». L'événement particulier auquel fait ici allusion Kacem et auquel la recommandation du Coran fait suite est le harcèlement que de jeunes désœuvrés, dans les rues de Médine, faisaient subir aux passantes qu'ils prenaient pour des esclaves bonnes à draguer (p. 52). 
18. On ne perdra pas non plus de vue l'impulsion qu'a donnée au succès de ce film l'actualité de la guerre en Afghanistan.

19. Ce traitement déséquilibré explique pourquoi des voix se sont élevées contre l'homme qui joue le rôle de Sahid, dont on n'a pas hésité à fouiller la vie privée. En effet, l'homme serait soupçonné d'être sur la liste des meurtriers américains et dénoncé comme un terroriste dangereux. Lorsqu'on a interrogé le réalisateur sur la vie passée de cet acteur, il a répondu qu'il choisit ses acteurs parmi les gens ordinaires dont il ne questionne ni le passé ni l'avenir: «I am an artist, not a judge, or a policeman, or an FBI agent. " Il dit qu'il n'aurait d'ailleurs aucun regret s'il s'avérait que Hasan Tantai, dans le rôle de Tahib Sahid, était un meurtrier, car il aurait, le cas échéant, le mérite de "have turned an American murderer into a reformist who regrets violence" (Hamid Dabashi, "Mohsen Makhmalbaf's Perspective Concerning the Character of Tabib Sahid in Kandahar", disponible en ligne à l'adresse suivante: $<$ http://www.makhmalbaf.com/articles.php?a=368 >).

20. Sur les articulations des cinématographies multiculturelles dans des espaces politiques multiculturels, on consultera Tropical Multiculturalism: A Comparative History of Race in Brasilian Cinema and Culture de Robert Stam (1997).

\section{RÉFÉRENCES BIBLIOGRAPHIQUES}

Bédard 1990 : Yves Bédard, «Images technologiques: ce qu'il advient de la mémoire», Cinémas, vol. 1, n 3, 1990, p. 89-101.

Boutot 1989: Alain Boutot, Heidegger, Paris, PUF, 1989.

Chevalier 1977 : Jean Chevalier (dir.), Dictionnaire des symboles. Mythes, rêves, coutumes, gestes, formes, figures, couleurs, nombres, Paris, Robert Laffont, 1977.

Cohan et Hark 1997 : Steven Cohan et Ina Rae Hark (dir.), The Road Movie Book, New York, Routledge, 1997.

Corrigan 1991 : Timothy Corrigan, A Cinema Without Walls: Movies and Culture After Vietnam, New Brunswick, Rutgers University Press, 1991.

Espagne 1999: Michel Espagne, Les transferts culturels franco-allemands, Paris, PUF, 1999.

Gaudreault et Marion 1994: André Gaudreault et Philippe Marion, «Dieu est l'auteur du documentaire...", Cinémas, vol. 4, n² 2, 1994, p. 11-26.

Geadah 1996 : Yolande Geadah, Femmes voilées. Intégrismes démasqués, Montréal, VLB, 1996.

Haghighat 2001 : Mamad Haghighat, "Entrevue avec Mohsen Makhmalbaf», livret à l'intérieur de la pochette de Kandahar, 2001.

Heidegger 1965: Martin Heidegger, L'Être et le Temps, Paris, Gallimard, 1965.

Heidegger 1967 : Martin Heidegger, Sein und Zeit, Tübingen, Max Niemeyer Verlag, 1967.

Heidegger 1997 : Martin Heidegger, Vom Wesen der Wahrheit, Frankfurt am Main, Klostermann, [1943] 1997.

Kacem 2004: Abdelaziz Kacem, Le voile est-il islamique? Montpellier, Chèvre-Feuille étoilée, 2004.

Levieux 2001 : Michèle Levieux, "Kandahar ou les premières images d'un pays sans images", Humanité hebdo, 12 et 13 mai 2001, p. 43.

Liandrat-Guigues 2005: Suzanne Liandrat-Guigues, Esthétique du mouvement cinématographique, Paris, Klincksieck, 2005. 
Loiselle 2005: Marie-Claude Loiselle, «Filmer l'autre», 24 Images, n 121, 2005, p. 9.

Macnab 2001: Geoffrey Macnab, "The Land Without Face», The Guardian, 11 aout 2001, p. 7.

Minces 1990 : Juliette Minces, La femme voilée. L'slam au féminin, Paris, CalmannLévy, 1990.

Ortiz 1940 : Fernando Ortiz, Contrapunteo cubano del tabaco y el azúcar, Havana, Jesús Montero, 1940.

Pazira 2001 : Nelofer Pazira, "Refuge in the Dust», livret à l'intérieur de la pochette de Kandahar, 2001.

Saadaoui 1982: Naoual el Saadaoui, La face cachée d'Ève. Les femmes dans le monde arabe, Paris, Éditions des femmes, 1982.

Schneider 1997 : Irmela Schneider, "Von der Vielsprachigkeit zur Kunst der Hybridation. Diskurse des Hybriden", dans Irmela Schneider et Christian W. Thomsen (dir.), Hybridkultur. Medien - Netze - Künste, Köln, Wienand, 1997, p. 13-58.

Stam 1997 : Robert Stam, Tropical Multiculturalism: A Comparative History of Race in Brasilian Cinema and Culture, Durban/London, Duke University Press, 1997.

Vanoye et Goliot-Lété 1992: Francis Vanoye et Anne Goliot-Lété, Précis d'analyse filmique, Paris, Nathan, 1992.

Vernet 1980 : Marc Vernet, "Flash-back», dans Jean Collet, Michel Marie et al., Lectures du film, Paris, Albatros, 1980, p. 96-99.

Vernet 1980a : Marc Vernet, "Personnage», dans Jean Collet, Michel Marie et al., Lectures du film, Paris, Albatros, 1980, p. 177-180.

Vogel 1977 : Amos Vogel, Le cinéma. Art subversif, Paris, Buchet-Chastel, 1977.

Weibel 2000 : Nadine B. Weibel, Par-delà le voile. Femmes d'islam en Europe, Bruxelles, Complexe, 2000.

Williams 1982: Mark Williams, Road Movies: The Complete Guide to Cinema on Wheels, London, Proteus, 1982.

\section{ABSTRACT}

\section{On the Road to Kandahar}

\section{Pierre Kadi Sossou}

The Iranian filmmaker Mohsen Makhmalbaf's film Kandahar (2001), a sort of intercultural road movie, is built around journeys, cultural encounters and the dual identity of the character Nafas. Taking as its starting point the search for a sister who needs to be saved in Kandahar, the film uses travel narration as an expedient to lead the viewer through the nooks and crannies of the Taliban's Afghanistan. On this road of cleavages between Afghan men and women, Nafas is constantly raising and lowering her veil, or burka, giving rise to questions about the act of unveiling in both the strict sense of the term-removing the burka - and the figurative-revealing Afghans'living conditions to the world. This gesture of unveiling is the symbol of a freedom that is contrasted with the canons of religious fundamentalism. 